\title{
Coal Mine Robot Binocular Vision Recognition System Based on Fuzzy Neural Network
}

\author{
C.C. Shang \\ Engineering Training Center \\ Xi'an University of Science and Technology \\ China
}

\author{
H.W. Ma \\ Engineering Training Center \\ Xi'an University of Science and Technology \\ China
}

\begin{abstract}
In general, coal mine detection robot has characteristics of a poor adaption to uncertain underground environment in the process of rescue. This paper proposed a binocular vision recognition system based on fuzzy neural network. The system in view of general fuzzy neural network, adopt self-organizing learning algorithms, and add fuzzy rules and membership function parameters to obtain an improved fuzzy neural network algorithm, which will reduce errors during the recognition process of coal mine detection robot. The simulation results and actual underground measurements show that the system has a higher accuracy and shorter respond time.
\end{abstract}

Keywords-fuzzy neural network; binocular vision; coal mine detection robot

\section{INTRODUCTION}

Since after the accident in coal mine, the collapse of the roadway usually makes the process of disaster rescue work almost impossible, the introduction of visual robot to rescue work can feedback the specific information about the accident site to rescuers at the first time, so as to prevent the occurrence of secondary accidents. It is an urgent must-be-solved issue in the process of study on coal mine rescue robot to make a visual robot travel to the target area successfully in the underground operations after the accident, how to use the machine vision's identifying ability to obtain position information for roadway obstacle and to make right decisions.

Fuzzy logic, data fusion, neural network and other artificial intelligence technology have a good application in the path planning with unknown environmental information, while the stereo vision technology also can remedy the uncertainty and incompleteness of environmental information collection for traditional sensors. Consequently, artificial intelligence methods based on stereo vision obstacle-avoiding strategies become a research focus in the field of robot path planning. For example, in terms of data fusion and fuzzy logic information technology, Lang et al[1] proposed mobile robot fuzzy path planning method for full coverage of the road. Moreover, Perez et al[2] suggested a path planning method based on fuzzy velocity field. In addition, Zun et al[3,4] proposed a mobile robot path planning and obstacle-avoiding method based on information fusion technology. In terms of neural network applications, Ghatee et al[5] proposed the use of Hopfield neural network optimization method for mobile robot path planning in the distance and security clearance. Furthermore, Zhu et al[6] applied the SOM self-organizing neural network to path planning of multi-task multi-robots. Practically, the foregoing discussion methods would mostly overcome the defects of many traditional planning methods, but there are still shortcomings. Especially, problems are mainly difficult to obtain samples in the self-learning process. In addition, a long time must be used for learning, and the system has a serious problem of hysteresis with poor real-time.

To solve these problems, this paper proposes an underground robot binocular vision obstacle recognition system based on fuzzy neural network, using five fuzzy neural networks, adopting self-organizing learning algorithms, and adding fuzzy rules and membership function to adjust and optimize the membership functions and get the desired output. Furthermore, MATLAB simulation and actual underground measurements show that the fuzzy neural network has a good adaptive capacity for the non-linear mapping, and data processing capability with a good real-time responding performance. Besides, the robot is of a good application effect in the process of moving in the coal mines.

\section{STRUCTURES AND ALGORITHMS OF FUZZY NEURAL NETWORK}

In this system, the fuzzy neural network is designed a five-layer structure shown in Figure 1, which also illustrated the basic functions of each layer of the network.

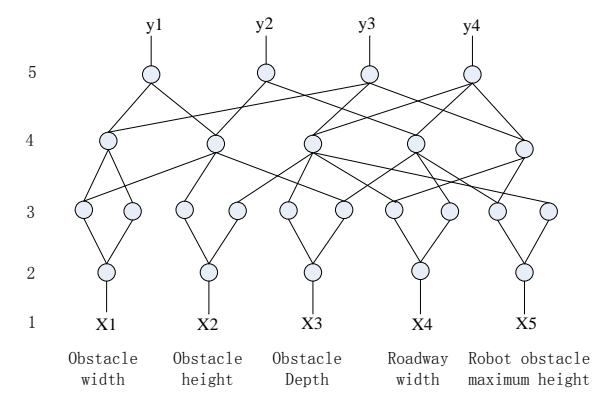

FIGURE I. FIVE LAYER FUZZY NEURAL NETWORK

The first layer also called the input layer. There are a total of five inputs.

In the second layer, the input is fuzzified and the input variable is calculated which belongs to the fuzzy membership degree of each fuzzy set[7]. Among them, the excitation function node is the premise of the membership function of each fuzzy rule of each fuzzy set. The output node of the second layer is: 


$$
O_{i}^{2}=\mu_{A_{i}}\left(x_{i}\right)
$$

The membership function is:

$$
\mu_{A_{k}}\left(X_{k}\right)=\min \left[\frac{1}{1+e^{-\sigma\left(X_{k}-v\right)}}, \frac{1}{1+e^{\sigma\left(X_{k}-v\right)}}\right]
$$

Where $\sigma$ represents the membership function center, $v$ represents membership function width.

In the third layer, accumulation reasoning is achieved. The node output is the product of the input and the output represents the degree of matching input node samples and rule premise $^{[7]}$. If the premise is the dimension of a feature article consists of $M$ feature, then the node output is:

$$
O_{i}^{3}=\prod_{j=1}^{M} O_{i}^{2}=\prod_{j=1}^{M} \mu_{x_{i}}
$$

In the fourth layer, implements center anti-fuzzy, specifically, the third layer collection weight $w_{i j}$ is center of the $\mathrm{i}$-th value of the output which is connected to the $\mathrm{j}$-th rule, an output node for the fourth layer is:

$$
O_{i}^{4}=\frac{\sum_{j=1}^{S} w_{i j} O_{i}^{3}}{\sum_{k=1}^{S} O_{k}^{3}}
$$

While $\mathrm{S}$ is the number of rules to generate the output.

In the fifth layer, completes normalized. If the hybrid model of fuzzy neural network is used to fit or function approximation problems, such as fuzzy control problem, then it is sufficient to more than 4 layers. However, in this system, each of the output values are to be in interval $[0,1]$, so it is necessary to process Sigmoid conversion for the output of the fourth layer, which it is done by the network's fifth layer. Of course, since this layer is the normalized layer, it is also possible to use other normalization methods, such as linear normalization methods are possible. So there is no essential difference[8]. The fifth layer's node output is:

$$
O_{i}^{5}=\frac{1}{1+e^{-O_{i}^{4}}}
$$

In this system, there are 4 outputs totally.

In the foregoing discussion, fuzzy neural network is quite rough for the effect of the target information classification, mainly because the input signal fuzzy neural network is unable to determine the membership function parameters, namely the center and width of the membership functions. In other words, membership function parameters choosing depends usually on the results of previous studies of the experience, which is the estimated value, thus resulting in the relatively rough classification results of fuzzy neural network.

\section{IMPROVED FUZZY NEURAL NETWORK}

In response to the shortcomings of rough fuzzy neural network, we propose a modified fuzzy neural network system which solves uncertainty difficult of membership function parameter selection in the traditional fuzzy neural network, making the response of neural network system more quickly and the output more accurate.

In order to determine the membership function parameters, the neural network learning methods must be firstly determined. The improved fuzzy network is a five-layer forward network, using the back propagation (BP) algorithm. The mean squared error is chosen as the error function:

$$
E=\frac{1}{2} \sum_{i=1}^{C}\left(y_{i}-O_{i}^{5}\right)^{2}
$$

In Equation 7, $y$ is the desired output, when the class sample is labeled $\mathrm{i}, y_{i}=1$, the remaining $y_{i}=0$, where $\mathrm{j} \neq \mathrm{i}$. The following is the error propagation process step by step.

In the fifth layer:

$$
\frac{\partial E}{\partial O_{i}^{4}}=-\left(y_{i}-O_{i}^{5}\right) O_{i}^{5}\left(1-O_{i}^{5}\right)
$$

In the fourth layer:

$$
\begin{gathered}
\frac{\partial E}{\partial O_{i}^{3}}=\sum_{i=1}^{C} \frac{\partial E}{\partial O_{i}^{4}} \frac{\partial O_{i}^{4}}{\partial O_{j}^{4}}=\sum_{i=1}^{C} \frac{\partial E}{\partial O_{i}^{4}} w_{i j} \frac{\sum_{k=1}^{S} O_{k}^{3}-O_{j}^{3}}{\sum_{k=1}^{S} O_{k}^{3}} \\
\frac{\partial E}{\partial w_{i j}}=\frac{\partial E}{\partial O_{i}^{4}} \frac{\partial O_{i}^{4}}{\partial w_{i j}}=\frac{\partial E}{\partial O_{i}^{4}} \frac{O_{i}^{3}}{\sum_{k=1}^{S} O_{k}^{3}}
\end{gathered}
$$

Suppose $\mathrm{m}$ is a node of the adjustable parameters, the general learning rule is:

$$
\Delta m \propto-\frac{\partial E}{\partial m}
$$

Adjusted formula for fuzzy sets center $w_{i j}$ of classification rules conclusion is as follows

$$
w_{i j}(t+1)=w_{i j}(t)-\eta \frac{\partial E}{\partial w_{i j}}
$$


While $\eta$ is the learning rate. In order to obtain learning rules, calculate $\partial \mathrm{E} / \partial \mathrm{y}$ layer by layer, simultaneously use the center and the width of the membership functions as an adjustment functions.

In the third layer:

$$
\frac{\partial E}{\partial O_{k}^{2}}=\frac{\partial E}{\partial O_{j}^{3}} \frac{\partial O_{j}^{3}}{\partial O_{k}^{2}}=\frac{\partial E}{\partial O_{j}^{3}} \prod_{l \neq k} O_{l}^{2}
$$

The second layer:

$$
\frac{\partial E}{\partial v}=\frac{\partial E}{\partial O_{k}^{2}} \frac{\partial O_{k}^{2}}{\partial v}
$$

Adjustment functions of $\nu$ and $\sigma$ are as follows

$$
\begin{aligned}
& v(t+1)=v(t)-\eta \frac{\partial E}{\partial v} \\
& \sigma(t+1)=\sigma(t)-\eta \frac{\partial E}{\partial \sigma}
\end{aligned}
$$

Since this modified fuzzy neural network design, the initial network structure and parameters are derived from the fuzzy rule-based system, which is relatively close to the optimal case. Therefore the training rate is faster and generally wouldn't fall into a local optimum. Of course, in order to improve efficiency, improved BP algorithm can also be used to spread. The improved fuzzy neural network can approximate any continuous function. Because the above fuzzy neural network-based model is a heuristic process, the final result is a near-optimal design, which is a distinctive feature of the intelligent information processing [9].

\section{SYSTEM PLATFORM AND THE EXPERIMENTAL RESULTS}

In the binocular vision obstacle recognition system of the coal mine detection robot, we use the existing mobile robot as a carrier, loaded the binocular stereo vision system to built a mine detection robot binocular vision system research platform for experimental study ${ }^{[10]}$. Hardware experimental platform of the system is shown in Figure 2.

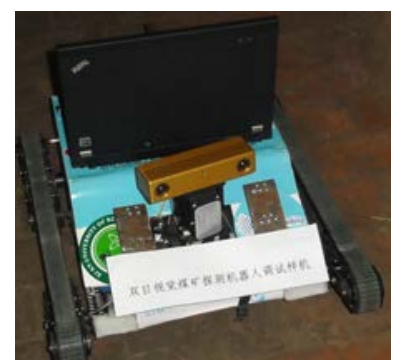

FIGURE II. THE SYSTEM HARDWARE PLATFORM

According to the maximum height which robot can travel across obstacles, there are four conditions for mine obstacles, namely: 1) no obstacles in front; 2) obstacles which robot can travel across in front; 3) there are insurmountable obstacles in front but can be bypassed; 4) there are insurmountable obstacles in front, and robot cannot go forward. Figure 3 shows the four types of obstacles.

For the visual obstacle-avoiding system software design, each fuzzy neural network system has five inputs and four outputs, namely, information on the horizontal width, height and depth of obstacle, roadway width as well as the maximum height for the robot through obstacles.

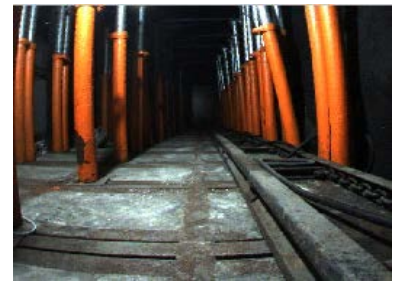

a. no obstacles

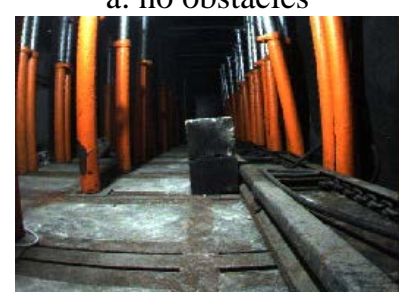

c. can bypass obstacles

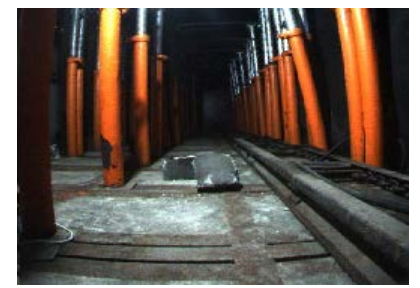

b. can cross obstacles

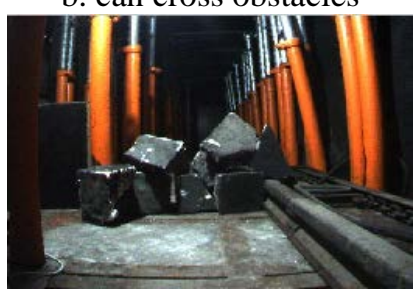

d. no forward

The network output is a four-dimensional vector whose desired output vector are: 1000 represents case a; 0100 indicates case b; 0010 indicates case b; 0001 indicates case d.

In this system, to choose the precise parameter for the fuzzy neural network, the first step is to operate the training in the fuzzy neural network. In order to do that, certain inputs and outputs from the system are needed. According to the actual situation of this paper, extraction three types of obstacles include: the obstacle shape features, the depth of the obstacle information and environmental information. Specifically, the obstacle shape features include the horizontal width, height information and the obstacle depth feature. The obstacle depth information was extracted according to the Binocular Vision system. Environmental information includes roadway width information and the robot through the maximum height of obstacle, wherein the width of roadway tunnel information and the robot through the maximum height of obstacle, wherein the width of roadway tunnel information will be getting to the recognition results of the extraction, and the robot through the maximum height information of obstacles ahead is given.

Experimental leaning object using 200 different types of obstacle images as training samples, including obstacle roadway pictures without obstacle (50 pictures), can be obstacle crossing roadway (50 pictures), do not cross the roadway but can bypass (50 pictures) and obstacle insurmountable and can not bypass tunnel (50 pictures). In addition, there is a picture of an unknown roadway 50 images as test sample. 
Firstly, correct the iteration of the neural network weight. Secondly, after the first round of the sample training finished, sum up the error signal and take the average. Thirdly, if the average error signal is larger than the present threshold value, then take the second round of training. Lastly, repeat the processes until the average error signal is smaller than the threshold value[11,12].

We use 200 unknown examples to conduct the MATLAB simulation. In the process, the absolute values of output which are smaller than 0.2 are considered as 0 . The absolute values of output in range of 0.8 to 1 are considered as 1 . Table 1 demonstrates part of the simulation results.

TABLEI. SIMULATION RESULTS OF PART SAMPLES

\begin{tabular}{|c|c|c|c|c|c|c|c|c|}
\hline $\begin{array}{c}\text { Sample } \\
\text { Number }\end{array}$ & \multicolumn{3}{|c|}{ Output } & \multicolumn{5}{c|}{ Actual Output } \\
\hline P0 & 1 & 0 & 0 & 0 & 0.997450 & 0.005152 & 0.006420 & 0.007827 \\
\hline P1 & 1 & 0 & 0 & 0 & 0.995870 & 0.004133 & 0.003231 & 0.006287 \\
\hline P2 & 0 & 1 & 0 & 0 & 0.003189 & 0.995621 & 0.005821 & 0.002781 \\
\hline P3 & 0 & 1 & 0 & 0 & 0.001321 & 0.998213 & 0.003721 & 0.004318 \\
\hline P4 & 0 & 0 & 1 & 0 & 0.003254 & 0.003128 & 0.998642 & 0.005632 \\
\hline P5 & 0 & 0 & 1 & 0 & 0.003621 & 0.004862 & 0.997532 & 0.006532 \\
\hline P6 & 0 & 0 & 1 & 0 & 0.003215 & 0.005328 & 0.999743 & 0.002716 \\
\hline P7 & 0 & 0 & 1 & 0 & 0.001742 & 0.005321 & 0.994387 & 0.003219 \\
\hline P8 & 0 & 0 & 0 & 1 & 0.005487 & 0.001287 & 0.003217 & 0.996732 \\
\hline P9 & 0 & 0 & 0 & 1 & 0.001275 & 0.004213 & 0.005821 & 0.993214 \\
\hline
\end{tabular}

Observed from table 1 , simulation result of the mine robot navigation decision making accuracy is $99.28 \%$. Moreover, the square root error of the improved mode of the fuzzy neural network simulation result is 7.2E-4, which proves that the simulation could determine whether there are obstacles in front or not in order to make a right decision.

In terms of responding time and error, fuzzy neural network has relative short response period with high accuracy. It also has good dynamic tracking and nonlinear approaching ability. In the simulation results shown in Figure 4, the improved model of this paper in terms of response time has a very excellent performance than the traditional neural network

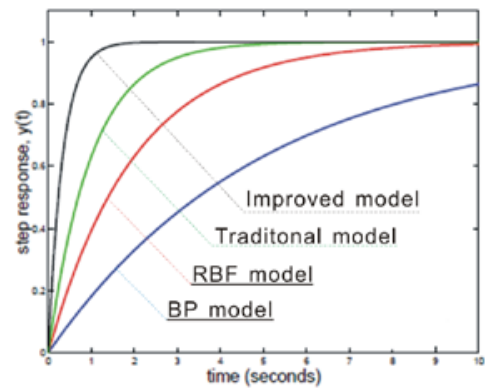

FIGURE IV.

FOUR TYPES OF NEURAL NETWORK SIMULATION

\section{CONCLUSIONS}

This paper presents an improved coal mine robot binocular vision recognition system based on fuzzy neural network. We apply the general structure of fuzzy neural network system [9], use self-organizing learning algorithms, and add fuzzy rules and parameter selection methods of membership functions, effectively improving the speed and accuracy of using fuzzy neural network for fuzzy classification of the collected obstacle information by the binocular vision. Simulation results show that the improved fuzzy neural network model has a strong adaptive ability for narrow underground mine environment with better system robustness, to ensure mobile robot's real-time obstacle-avoiding performance in an uncertain environment. Taking our self-developed coal mine caterpillar track robot as carrier, field test shows that the system has better characteristics of good real-time and high accuracy.

\section{REFERENCE}

[1] Fu Y, Lang S Y L. Fuzzy logic based mobile robot area filling with vision system for indoor environment[C]. IEEE Int Conf on Computational Intelligence in Robotics and Automation. Monterey, 1999: 326-331.

[2] Perez D A, Melendez W M, Guzman J, et al. Fuzzy logic based speed planning for autonomous navigation under velocity field control[C] IEEE Int Conf on Mechatronics. Malaga, 2009: 14-17.

[3] Zun A D, Kato N, Nomura Y, et al. Path planning based on geographical features information for an autonomous mobile robot[J]. Artificial Life and Robotics, 2006, 10(2): 149-156.

[4] Shen D, Chen G S, Cruz J J, et al. A game theoretic data fusion aided path planning approach for cooperative UAV ISR[C]. IEEE Int Conf on Aerospace. Montana, 2008:1-9.

[5] Ghatee M, Mohades A. Motion planning in order to optimize the length and clearance applying a Hopfield neural network[J]. Expert Systems with Applications, 2009, 36: 4688-4695.

[6] Zhu A, Yang S X. A neural network approach to task assignment of multi-robots[J]. IEEE Trans on Neural Network, 2006, 17(5): 1278-1287.

[7] Lin Cheng-Jian, Wang Jun-Gao, Lee Chi YUNG. Pattern recognition using neural fuzzy networks based on improved particle swam optimization [J]. Expert System with Applications, 2008(6):1-9.

[8] Marical G N, Toledo J, Acosta L, et al. A neuron-fuzzy method applied to the motors of a stereo vision system [J]. Engineering Applications of Artificial Intelligence, 2007(4):951-985.

[9] Yang J,Sun H, Wu L. Application of fuzzy neural networks in information Fusion for obstacle avoidance[J]. Techniques of Automation and Application 2005(02) : 276-282.

[10] Shang Changchun, Ma Hongwei, Zhang Qi, Coal Mine Detection Robot Inhence Vision Technology Research [J]. Computer Mesurement and Control,2013.21(10) : 2777-2779.

[11] Li Weiqing, Wang Chengbiao, Wang Qun,et al. An edge detection method based optimized BP neural network[J]. Internatioal Syniposium on Informatin Science and Engineering,2008(2):40-44.

[12] Enrico Zio, Giulio Gola. A neuron-fuzzy technique for fault diagnosis and its application to rotating machinery[J]. Reliability Engineering and System Safety, 2007(5):78-88. 